
extravasation.

Others have pushed for early catheter removal but have admitted a higher chance of urinary

I think what is clear from this article is that surgeons have a choice - they can leave the catheter in for a long period of time and not perform imaging or they can try to remove the catheter early but should perform imaging if they do so. With prolonged catheterization comes increased risk of urinary tract infection and patient discomfort. With early catheter removal comes the risk that about 1 in 5 will need the catheter replaced for another week or two due to extravasation on imaging. It would seem that for patients who live close to a reconstructive center, early catheter removal with imaging and a chance of catheter replacement may be preferable. For those who live far from their reconstructive surgeon or who want to minimize extra visits, a longer period of catheterization without subsequent imaging would be appropriate.

Dr. Sean P. Elliott

Department of Urology Surgery

University of Minnesota

Minneapolis, Minnesota, USA

E-mail: selliott@umn.edu

\title{
Long-term results of permanent urethral stent Memotherm implantation in the management of recurrent bulbar urethral stenosis
}

Sertcelik MN, Bozkurt IH, Yalcinkaya F, Zengin K

Ankara Diskapi Yildirim Beyazit Training and Research Hospital 1. Urology Clinic, Ankara, and

Department of Urology, Karaman State Hospital, Karaman, Turkey

BJU Int. 2011; 108: 1839-42. doi: 10.1111/j.1464-410X.2011.10230.x.

Study Type - Therapy (case series) Level of Evidence4 What's known on the subject? and What does the study add? Milroy reported $84 \%$ success at a mean of 4.5 years follow-up by usage of a permanently implantable "urolume" spent in 1993. Study Type - Therapy (case series) Level of Evidence Memotherm was developed later, especially for urologic use. Our study is one of the largest in this urea, with a high number of patients and a long follow-up period.

Objective: To evaluate the effectiveness and long-term results of permanent urethral stent (Memotherm) implantation in the treatment of recurrent bulbar urethral stricture.

Patients and Methods: In all, 47 patients with a history of previous unsuccessful treatment for bulbar urethral stricture were treated using Memotherm bulbar urethral stents between 1998 and 2002. Long-term follow-up data was analysed and discussed.

Results: At the end of the 7-year period 37 of 47 patients $(78.7 \%)$ had been treated successfully. Post-micturition dribbling incontinence lasting up to 3 months after stent placement occurred in $32(68.1 \%)$ patients, but this was reduced to only seven patients $(14.9 \%)$ by the 7-year follow-up. There was stress incontinence of various severities in nine (19.2\%) patients at the 1-year follow-up. These patients were those who had stenosed urethral segments adjacent to the external sphincter. At the long-term follow-up $<10 \%$ of the patients had stress incontinence complaints.

Conclusion: Memotherm is a good treatment option in patients with recurrent bulbar urethral stricture of any cause. 


\title{
Editorial Comment
}

We do not yet have a urethral stent that is ready for broad application. The authors describe their experience with Memotherm. This is a stent that contracts in cold water and expands after being placed in the urethra at body temperature. Removing it is fairly easy after irrigating the urethra with cold water. This is a big improvement over the Urolume stent which is very difficult to extract. Still the results reported herein show that there is still much room for improvement in the design of urethral stents, or that stents hold limited promise in urethral stricture management. Approximately half of the patients required secondary procedures for restenosis or "hyperplastic ingrowth" inside the stent. The true future of stents likely lies in drug-eluting stents or temporary stents acting as scaffolds for engineered tissue ingrowth.

Dr. Sean P. Elliott

Department of Urology Surgery

University of Minnesota

Minneapolis, Minnesota, USA

E-mail: selliott@umn.edu

NEUROLOGY \& FEMALE UROLOGY

\author{
Long-term subjective results of tension-free vaginal tape operation for female urinary stress incontinence \\ Glavind K, Glavind E, Fenger-Grøn M \\ Department of Gynecology and Obstetrics, Aalborg Hospital, 9000, Aalborg, Denmark \\ Int Urogynecol J. 2011; 18. [Epub ahead of print]
}

Introduction and Hypothesis: The aim of the study was to evaluate the subjective outcome between 1 and 5 years after tension-free vaginal tape (TVT) operation and the need for follow-up.

Methods: A prospective questionnaire study was performed including questions about incontinence, urinary tract infection, emptying problems, the wish for a clinical control and the International Consultation on Incontinence Questionnaire-Short Form (ICIQ-SF).

Results: One hundred seventy-three patients were included. There were more patients with subjective recurrent stress incontinence over the years, but ICIQ-SF was unchanged. There was no rise in patients reporting urge incontinence over the years. Only $11.4 \%$ of the patients wished for a clinical control at some time.

Conclusion: The TVT operation showed a slight degree of subjective deterioration between 1 and 5 years after the operation; however, the ICIQ-SF was unchanged. There seems to be no need for long-term follow-up at the operating department.

\section{Editorial Comment}

This is an interesting paper by Glavind et al. aiming to show sustained results after TVT operation for treating female stress urinary incontinence (SUI) from 3 months up to 5 years of follow-up. Their idea is to question the need for such long follow-up.

They analyzed 173 patients and present an increase from $12.2 \%$ to $26.7 \%$ in recurrent SUI comparing 3 mo x 5 years results with an odds ratio (OR) of 1.38. A similar finding occurred for subjective complaints regarding difficulty to empty the bladder $(8.6 \%$ to $26.7 \%$ for 3 mo and 5 years, respectively, with a yearly OR of 1.57). However, the drop out rate of $38 \%$ during long term follow-up seems unacceptably high $(81 \times 51$ patients at 3 mo and 5 years). Despite this it is interesting to note that ICIQ-SF scores did not rise throughout time and only $11 \%$ of patients requested a clinical control over time. The authors conclude suggesting no need 